\title{
The Nucleotide Sequence of the LPD1 Gene Encoding Lipoamide Dehydrogenase in Saccharomyces cerevisiae: Comparison between Eukaryotic and Prokaryotic Sequences for Related Enzymes and Identification of Potential Upstream Control Sites
}

\author{
By JOE ROSS, * GRAEME A. REID AND IAN W. DAWES \\ Department of Microbiology, University of Edinburgh, West Mains Road, \\ Edinburgh EH9 $3 J G, U K$
}

(Received 30 November 1987; revised 29 January 1988)

\begin{abstract}
The complete nucleotide sequence of the LPDI gene, which encodes the lipoamide dehydrogenase component (E3) of the pyruvate dehydrogenase and 2-oxoglutarate dehydrogenase multienzyme complexes of Saccharomyces cerevisiae, has been established. The flanking region 5' to the $L P D 1$ gene contains DNA sequences which show homology to known control sites found upstream of other yeast genes. The primary structure of the protein, determined from the DNA sequence, shows strong homology to a group of flavoproteins including Escherichia coli lipoamide dehydrogenase and pig heart lipoamide dehydrogenase. The amino acid sequence also reveals the presence of a potential targeting sequence at its $\mathrm{N}$-terminus which may facilitate transport to and entry into mitochondria.
\end{abstract}

\section{INTRODUCTION}

Lipoamide dehydrogenase (EC 1.8.1.4) is a component of the multienzyme complexes pyruvate dehydrogenase and 2-oxoglutarate dehydrogenase, which catalyse the oxidative decarboxylation of pyruvate and 2-oxoglutarate to acetyl-CoA and succinyl-CoA respectively (Reed, 1974). Both complexes contain multiple copies of three component enzymes: pyruvate dehydrogenase (EC 1.2.4.1) or 2-oxoglutarate dehydrogenase (EC 1.2.4.2) (E1); dihydrolipoamide acetyltransferase (EC 2.3.1.12) or dihydrolipoamide succinyltransferase (EC 2.3.1.61) (E2) and lipoamide dehydrogenase (E3). The E1 and E2 components are specific to their respective complexes whereas lipoamide dehydrogenase has been shown to be functionally interchangeable between both (Mukherjee et al., 1965), and in Escherichia coli and Saccharomyces cerevisiae is encoded by a single gene (Guest \& Creaghan, 1973; Dickinson et al., 1986).

In higher eukaryotes lipoamide dehydrogenase has an additional role in the multienzyme complexes which specificially catalyse the oxidative decarboxylation of branched-chain 2oxoacids derived from leucine, valine and isoleucine by transamination (Lawson et al., 1983). The reversible oxidative decarboxylation of glycine has also been shown to involve lipoamide dehydrogenase in the aerobic bacterium Arthrobacter globiformis (Kochi \& Kikuchi, 1976), in the anaerobe Peptococcus glycinophilus (Robinson et al., 1973) and in rat liver mitochondria (Kochi \& Kikuchi, 1976).

Regulation of the synthesis of the E1, E2 and E3 components of pyruvate dehydrogenase and 2-oxoglutarate dehydrogenase in $E$. coli has been studied extensively (Guest \& Rice, 1984; Spencer \& Guest, 1985), and it has been shown that the $E$ and $F$ genes of the ace operon and the $A$ and $B$ genes of the suc operon encode the E1 and E2 subunits of the pyruvate dehydrogenase and 2-oxoglutarate dehydrogenase complexes respectively. Lipoamide dehydrogenase is encoded by a single gene, $l p d$, which is linked to the ace operon but can operate under the control of its own promoter. Transcription from $\operatorname{sucAB}$, aceEF and $l p d$ genes shows differential 
regulation in response to the nature of the carbon source, thereby ensuring the necessary ratio of subunit components when pyruvate and/or 2-oxoglutarate dehydrogenase enzyme complexes are required. The absence of operons in eukaryotic organisms dictates that the regulation of synthesis of the E1, E2 and E3 components in yeast must differ from the system found in E. coli.

The role of lipoamide dehydrogenase in at least two multienzyme complexes raises several interesting questions concerning the regulation of synthesis and control of distribution of an enzyme which functions within two different complexes. The enzyme is encoded in the nucleus but must be transported to its site of action in the mitochondrion. Biochemical studies of the initiation of sporulation in $S$. cerevisiae have shown that the regulation of 2-oxoglutarate dehydrogenase activity may be of crucial importance in the switch of cells from vegetative growth via mitosis to the developmental processes of meiosis and sporulation (Dickinson et al., 1986). In $S$. cerevisiae the enzyme has also been shown to be subject to catabolite repression (Roy \& Dawes, 1987).

Determination of the DNA sequence for the genes which encode the E1, E2 and E3 components of both pyruvate and 2-oxoglutarate dehydrogenase is necessary in order to study how their syntheses are regulated at the molecular level.

\section{METHODS}

Sources of DNA. The $3.7 \mathrm{~kb}$ XhoI fragment which contains the LPD1 gene was obtained from pGP-R1 (Roy \& Dawes, 1987) for 'shotgun' cloning into M13 vectors. Large-scale plasmid and phage RF2 DNA isolation was performed by alkaline-SDS lysis; the DNA was purified by centrifugation in $\mathrm{CsCl} /$ ethidium bromide gradients (Maniatis et al., 1982). Restriction fragments were separated by electrophoresis in agarose gels and extracted by electro-elution onto dialysis membrane (Smith, 1980).

Cloning in the M13-based vector. The $3.7 \mathrm{~kb}$ XhoI fragment was digested with three restriction enzymes for shotgun cloning into M13mp18 (Messing, 1983). TaqI and MspI fragments were cloned into the AccI site of M13mp18, and Sau3AI fragments were cloned into the BamHI site of the same vector. In addition, the $1.45 \mathrm{~kb}$ $X b a I$ fragment was cloned into the $X b a I$ site of M13mp18. Transfection of E. coli JM101 [ $\Delta($ lac-pro) supE thi/F' traD36 proAB lacI $\left.{ }^{4} Z \Delta M 15\right]$ was performed according to published procedures (Winter \& Fields, 1980).

Nucleotide sequence analysis. Single-stranded M13 DNA templates were prepared and sequenced by the dideoxy chain-termination method using a 17-nucleotide synthetic primer (Sanger et al., 1977). Two 15-nucleotide synthetic primers, based on information obtained from the LPDI sequence, were also used. The nucleotide sequence was compiled and analysed using the University of Wisconsin Genetics Computer Group programs (Devereux et al., 1984).

Materials. Restriction enzymes were purchased from Bethesda Research Laboratories, Boehringer, and Northumbria Biologicals. Phage T4 DNA ligase was from either Boehringer or Northumbria Biologicals. The 17nucleotide synthetic primer was obtained from Pharmacia; the 15-nucleotide synthetic primers were purchased from OSWEL DNA Synthesis Service, Chemistry Department, Edinburgh University. DNA polymerase (Klenow fragment), dideoxynucleotides and deoxynucleotides were obtained from Boehringer, $\left[\alpha^{-32}\right.$ P]dATP from Amersham, and reverse transcriptase from Northumbria Biologicals.

\section{RESULTS AND DISCUSSION}

\section{The nucleotide sequence}

Based on deletion analysis of the plasmid pGP1 (Roy \& Dawes, 1987), a YEpl3-based vector containing a $5.5 \mathrm{~kb}$ yeast DNA insert carrying the $L P D I$ gene, a $3.7 \mathrm{~kb}$ Xhol fragment was chosen for 'shotgun' cloning analysis. A region of $2701 \mathrm{bp}$ has been sequenced. All of the sequence was obtained from at least two independent clones, it was fully overlapping and was, with the exception of a $0.1 \mathrm{~kb}$ region $0.8 \mathrm{~kb}$ upstream of the coding region, derived from both DNA strands. The program MAP was used to identify the coding region representing the $L P D 1$ gene. One large open reading frame of $1.5 \mathrm{~kb}$ was found, which is consistent with the known size of the yeast lipoamide dehydrogenase subunit polypeptide (Wieland, 1983) and which exhibits a consistently high score with respect to preferred codon usage in $S$. cerevisiae determined using the programs CODONFREQUENCY and CODONPREFERENCE (data not shown). The complete and unambiguous sequence of the LPDI gene and the primary structure of lipoamide dehydrogenase, translated from the DNA sequence, are presented in Fig. 1. 
In the region 5 -distal to the $L P D I$ sequence are two potential open reading frames: one (at -571), which would be read in the opposite sense, is capable of encoding a polypeptide of 77 residues; the other (sequence not complete, terminating at -858) could encode a polypeptide of at least 76 amino acids. A search of the NBRF protein data base showed no strong homology between the two predicted amino acid sequences and any known protein.

\section{Primary structure of yeast lipoamide dehydrogenase}

The primary structure, translated from the nucleotide sequence of the LPDI gene, contains 499 amino acid residues that correspond to a protein of $M_{\mathrm{r}} 54010$ (54730 including the FAD cofactor). These $M_{\mathrm{r}}$ values are in good agreement with previous estimates (Wieland, 1983).

Lipoamide dehydrogenase, encoded in the nucleus and synthesized on cytoplasmic ribosomes, is a component of two multienzyme complexes which function in the mitochondrial matrix-inner membrane compartment. The presence of an $\mathrm{N}$-terminal targeting sequence, which directs the protein to its correct subcellular location and facilitates its entry into the mitochondrion, has been shown in mammalian cells. Antibodies raised against the E1, E2 and E3 components of mitochondrial 2-oxoglutarate dehydrogenase from ox heart have been used to show the presence, in cultured pig kidney cells, of initial cytoplasmic translation products that are larger than the mature proteins (Hunter \& Lindsay, 1986). The E3 component is synthesized as a polypeptide $10-20$ amino acids larger than the mature protein. The primary structure, derived from the $L P D 1$ nucleotide sequence of $S$. cerevisiae, supports the conclusion that, in this organism, the lipoamide dehydrogenase $\mathrm{N}$-terminal region represents a mitochondrial targeting sequence. The first 20 amino acids are rich in seryl, threonyl and basic residues, and show a complete absence of acidic residues. All of these features are typical of mitochondrial targeting sequences in yeast (von Heijne, 1986). In $E$. coli such a targeting sequence would be unnecessary; and when the primary structures of $E$. coli and $S$. cerevisiae lipoamide dehydrogenase (Stephens et al., 1983) are aligned (see Fig. 2) the yeast sequence is seen to contain an additional 20 amino acids at its $\mathrm{N}$-terminus which are absent from the inferred $E$. coli protein.

The primary structures of several flavoproteins have been determined either partially or completely. Pig heart lipoamide dehydrogenase, E. coli lipoamide dehydrogenase, human erythrocyte glutathione reductase and Pseudomonas aeruginosa transposon mercuric reductase amino acid sequences have previously been compared (Williams et al., 1984) and four regions were identified which show strong homology between the four proteins. Two of these (FAD-1 and FAD-2) are involved in the binding of FAD, and the others constitute a pyridine nucleotide binding domain and an 'interface' domain involved in the interaction between subunits of the enzyme. Fig. 2 shows the primary structure of these four proteins and of $S$. cerevisiae lipoamide dehydrogenase aligned for maximum homology using the computer programs GAP and PRETTY. The primary structure of $S$. cerevisiae lipoamide dehydrogenase is in full agreement with the established 'domain homology' found between the other proteins. Comparison of the three lipoamide dehydrogenase primary structures reveals that the strongest homology lies between those of pig heart and yeast. The higher level of homology is particularly noticeable in the dimer interface region concerned with the interaction of the two polypeptides in formation of the active dimeric form (see Fig. 2). This may indicate that there are differences between prokaryotes and eukaryotes in the recognition of the subunits during assembly, which is reflected in the different organization of the multienzyme complexes in terms of subunit composition, especially for pyruvate dehydrogenase (Reed, 1974). Despite these differences the overall degree of homology that exists between all three polypeptides indicates the heavy evolutionary constraints placed upon an enzyme which functions within two different multienzyme complexes. This is also reflected in the low immunogenicity of the yeast E3 subunit relative to the other enzymes of the pyruvate and 2-oxoglutarate dehydrogenase complexes (De Marcucci et al., 1985).

\section{General features of the noncoding regions}

The sequences upstream and downstream which flank the coding region of the $L P D 1$ gene share several characteristics common to the $5^{\prime}$ - and $3^{\prime}$-noncoding regions of many sequenced yeast genes. 
GCAAGCTCTTGATTGATCAATIGGTAACTATCGGCTTIGACTACGGTTGTTATAMGATIATITTAGATTGCGATGAGAMAATGTCAMITCTATGAAM $-1000$ ATGTGGGTTAGCAACGCAGGCGTGGAMTGCAAATTAGAMATAGTATTGCTGTATAGTACATAGAGGTCGACATATATATATATATATATMTTTTAT $-900$

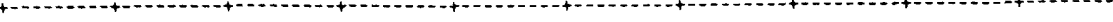
ATGGCATCTAGAGCCTCTAGGGATATTATGGAGTTGCCCCTAATGACGGTCTGCAAGCCTAGCTGGTGGTIATTGGCAGGGTCTTCACCATTIATCTCCA -800 +-2 TTGCATCATCAAGAMCGACGTTTAMGMUATATCGTAGCCTCGCAMATTCCTGCCACTTICCTAGATCCATTTATATTCAGCAMTATCTICTTGTCCAT -700 + ATATTTCTTCAGTTCAGGGGTAGAACCATTGTATCTGTGCGTGTGTITGCGTAATCCTGTCCTATGCCGGATACTCTATATGCGTTGTACTTTCTTATA CATTCATTGCCACCTITAGAGATTTAMTITTCTTACCACATTGTITCTATCAGCACAAACGTGAMGAGCGTAGGCAGGCTATAGGMMAAAGGGAMI GAGGACAGAGGAMTATCGAACAMTGCCACTGACAAACGTATGCGTGACTACEACGMTAGTCATGATGCAATGTACCCTCATATATCCCACTAMAMTA

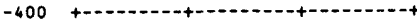
TATACTTACTAMGGTTTATGCACGTGATATCACGTGATCGTTTT TAM GATGACTCGTTTT TAGATACTTTATCATCTCGACGGCTGTTCCCTCA

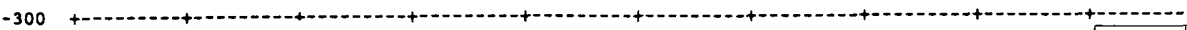

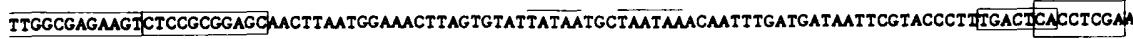

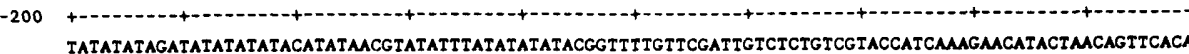

Met Leu Arg Ile Arg Ser Leu Leu Asn Asn lys Arg Ala Phe Ser Ser Thr Val Arg Thr Leu Thr Ile Asn Lys ATG TIA AGA ATC AGA TCA CTC CTA MT AMT MG CGT GCC TTT TCG TCC ACA GTC AGG ACA TTC ACC ATT MAC AG

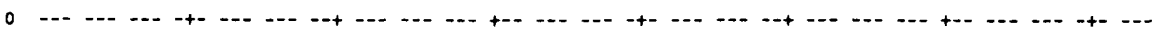
Ser His Asp Val Val Ile Ile Gly Gly Gly Pro Ala Gly Tyr Val Ala Ala Ile Lys Ala Ala Gin Leu Gly Phe TCA CAT GAT GTA GTC ATC ATC GGT GGT GGC CCT GCT GGT TAC GTG GCT GCT ATC MA GCT GCT CA ITG GGA ITT 75 Asn Thr Ala Cys Val Glu Lys Arg Gly Lys Leu Gly Gly thr Cys Leu Asn Val Gly Cys Ile Pro Ser Lys Ala MAC ACT GCA TGT GTA GM AMA AGA GGC MA TTA GGC GGT ACC TGT CTT AAC GTT GGA TGT ATC CCC TCC MA GCA

150 + Leu Leu Asn Asn Ser Bls Leu Phe His Gln Met HLs The Glu Ale Gln Lys Arg Gly Ile Asp Val Asn Gly Asp CTT CTA MI MAT ICT CAT ITA ITC CAC CAN ATG CAT ACG GA GCG CAA MG AGA GGT ATT GAC GTC MAC GGT GAT

225 -... - +... - Ile Lys Ile Asn Val Ale Asn Phe Gln Lys Ala Lys Asp Asp Ala Val Lys Gln Leu Thr Gly Giy Ile Glu Leu ATC AMA ATT AAC GTA GCA MAC TTC CAN MAG GCT AAG GAT GAC GCT GIT AAG CAA TIA ACT GGA GGT ATT GAG CTT tou CTG TTC AAG AAA AAT MAG GTC ACC TAT TAT AAA GGT AMT GGT TCA ITC GAA GAC GA ACG MG ATC AGA GTA ACT

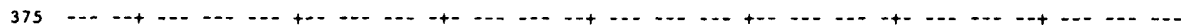
Pro Val Asp Gly Leu Glu Gly Thr Val Lys Glu Asp His Ile Leu Asp Val Lys Asn Ile Ile Val Ala Thr Gly CCC GTI GAT GGG ITG GM GGC ACT GTC AAG GAA GAC CAC ATA CTA GAT GTT AAG AAC ATC ATA GTC GCC ACG GGC

450 + Ser Glu Val Thr Pro Phe Pro gly Ile Glu Ile Asp Glu Glu Lys Ile Val Ser Ser Thr Gly ale Leu Ser Leu TCT GAA GTT ACA CCC TTC CCC GGT ATT GAA ATA GAT GAG GAA AAA ATT GTC TCI ICA ACA GGT GCT CTT TCG TTA

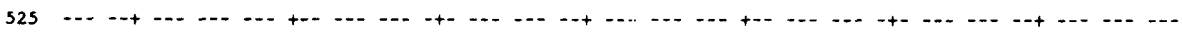
Lys Glu Ile Pro Lys arg Leu Thr Ile Ile Gly Gly Gly Ile Ile Gly Leu Glu Met Gly Ser Val Tyr Ser arg AAG GA ATT CCC AMA AGA TTA ACC ATC ATT GGT GGA GGA ATC ATC GGA TTG GAA ATG GGT TCA GTT TAC TCT AGA

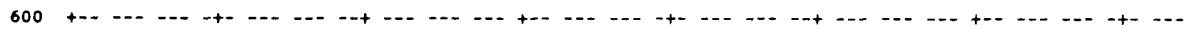
Leu Gly Ser Lys Val thr Val Val Glu Phe Gln Pro Gln Ile Gly Ala Ser Met Asp Gig Glu Val Ala Lys Ala ITA GGC TCC MAG GTT ACT GTA GTA GM TTT CAA CCT CAA ATT GGT GCA ICT ATG GAC GGC GAG GTT GCC AAA GCC

675 Thr Gln Lys Phe Lou Lys Lys Gln Gly Leu Asp Phe Lys Leu Ser Thr Lys Val Ile Ser Ala Lys Arg Asn Asp ACC CAN AAG ITC TTG AMA MG CM GGT ITG GAC TTC MA ITA AGC ACC MA GTI ATT TCT GCA MG AGA MAC GAC

750 + Asp Lys Asn Val Val Glu Ile Val Val Glu Asp Thr Lys Thr Asn Lys Gin Glu Asn Leu Glu Ala Glu Val Leu GAC MAG MAC GTC GTC GM ATT GTT GTA GA GAT ACT AMA ACG AAT AAG CAA GAA MAT TTG GA GCT GAA GTT TTG

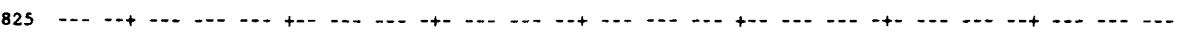
Leu Val Ala Val Gly Arg Arg Pro Tyr Ile Ala Gly Leu Gly Ala Glu bys Ile Gly leu Glu Val Asp Lys Arg CTG GIT GCT GIT GGT AGA AGA CCT TAC ATT GCT GGC TTA GGG GCT GAA AAG ATT GGA ITA GA GTA GAC AMA AGG

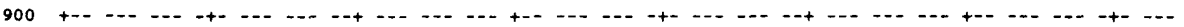
Gly Arg Leu Val Ile Asp Asp Gin Phe Asn Ser Lys Phe Pro his Ile Lys Val Val Giy Asp Val Thr Phe Gly GGA CGC CTA GTC ATT GAT GAC CAA ITT AAT TCC AAG ITC CCA CAC ATT AAA GTG GTA GGA GAT GTT ACA ITT GGT 


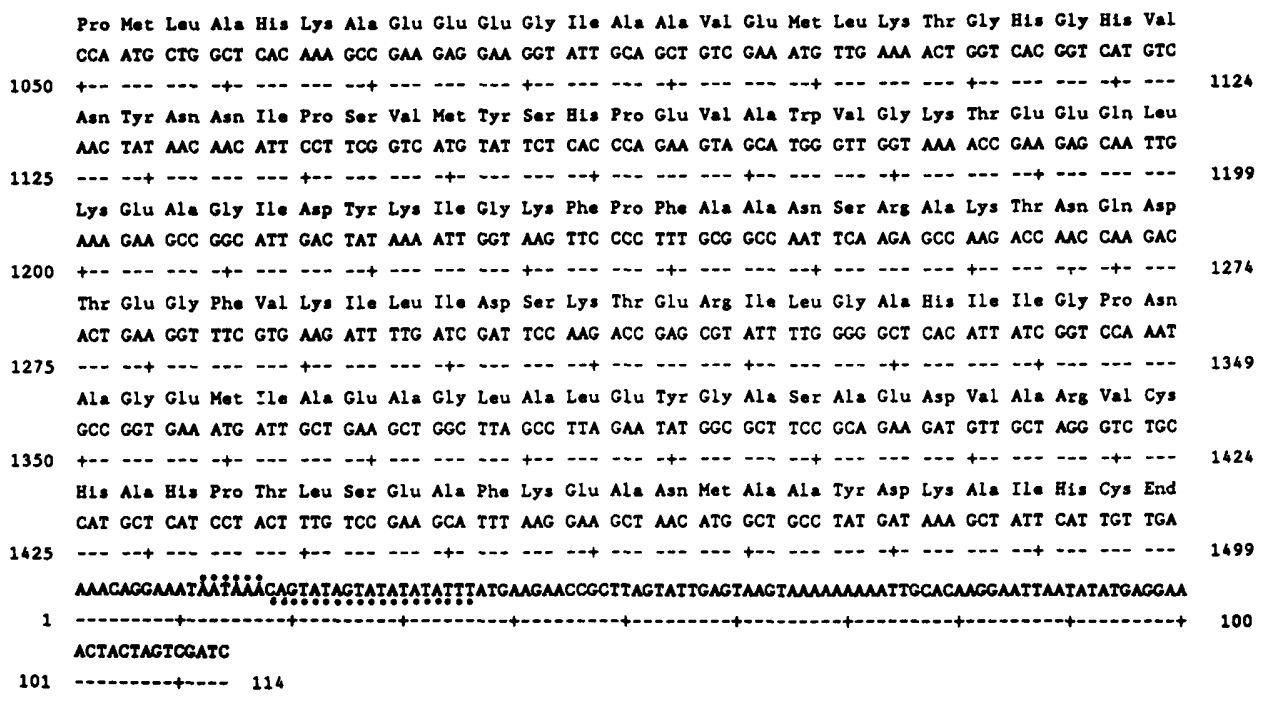

Fig. 1. Nucleotide sequence of the $L P D I$ gene and deduced primary structure of lipoamide dehydrogenase. The nucleotide sequence of a $2 \cdot 7 \mathrm{~kb}$ region containing the sense strand of the $L P D 1$ gene as well as over $1 \mathrm{~kb}$ of flanking DNA is shown in the $5^{\prime}$ to $3^{\prime}$ direction. The specific features of the 5 ' flanking region are noted as follows: The 'TATA' consensus sequences are overlined; the sequences which show homology to known regulatory sequences are boxed. Specific features of the $3^{\prime}$ flanking sequence are noted as follows: the proposed consensus polyadenylation signal, AATAAA, is denoted by overdots; the proposed polyadenylation/termination signal TAG...TATGT...TTT is denoted by underdots.

The noncoding regions of several yeast genes are AT rich and the regions $5^{\prime}(-500$ to -1$)$ and $3^{\prime}$ to the $L P D 1$ coding region have overall A + T compositions of $63 \%$ and $74 \%$ respectively. The sequences TAATAA and TATAA found at positions -146 and -154 represent potential TATA boxes believed to be important in positioning transcription initiation by RNA polymerase II (Grosschedl \& Birnstiel, 1980). An A is found at position -3 as has been reported for the majority of yeast genes sequenced (Kozak, 1981).

Downstream of the TGA translation termination codon an additional 114 bp has been sequenced. The motif AATAAA postulated to specify a site involved in polyadenylation in higher eukaryotes (Proudfoot \& Brownlee, 1976) and seen in many yeast genes is found at position +12 . The sequence TAG...TA(T)GT ...(AT rich)... TTT has been postulated to be a signal for transcription termination or polyadenylation (Zaret \& Sherman, 1982), and a version of this is found at position +18: CAGTATAGTATATATATTT.

\section{Regulation of the LPDI gene}

The upstream region from -358 to -102 contains a number of motifs which show homology to ones that have roles in the transcriptional regulation of other yeast genes. This region therefore probably contains elements which control the expression of the LPDI gene.

These motifs are illustrated in Fig. 3. At position -247 there is a TGACTC sequence with an adjacent run of $\mathrm{T}$ residues that conforms very closely to the consensus sequence obtained from the GCN4 protein binding sites studied to date (Hill et al., 1986). This protein mediates the general control of amino acid biosynthesis response that is involved in modulating during amino acid starvation the expression of some genes for amino acid synthesis (Struhl, 1982). It is interesting that a second, almost perfect direct repeat sequence (TGAATCGTTTTT), is also present $17 \mathrm{bp}$ away at position -265 . At -114 there is another TGACTC motif, although this conforms less well to the GCN4 consensus. All other yeast genes found so far to be subject to this GCN4-mediated control are concerned directly with synthesis of amino acids or charged tRNA species. Regulation of lipoamide dehydrogenase by general amino acid control may be less 
1

YLPDH mlrirsllnn krafsstvrt 1tinkshDVV IIGGGPaGYv AAikAAqLGF nTacVEkrgk LGGTCLNVGC PLPDH $\ldots \ldots \ldots \ldots \ldots \ldots \ldots$ adqpidaDVt VIGsGPGGYv AAikAAqLGF kTVcIEkneT LGGTCLNVGC ECLPDH $\ldots \ldots \ldots \ldots \ldots \ldots \ldots \ldots$. steiktqVV VIGaGPaGYs AAfrCAdLGL eTViVErynT LGGVCLNVGC HGR $\quad . .$. acrq epqpqgpdpa agavasyDyl VIGGGsGG1a sArrAAeLGa raavVE.shk LGGTCvNVGC PAMR adnrvglldk vrgwmaaaek hsgneppvqV aViGsgGaam AAalkAveqg aqVtliergT iGGTCvNVGC

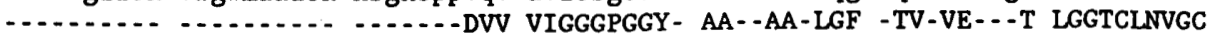

IPSKALLNns H1fHqMhtea qkrgi.dvng DIKiNvanfq kakDdaVkqL TGGie1LFKk nkVtyykGng

000

139

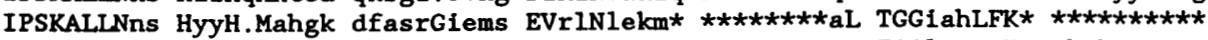
IPSKALL. . . HvakvieEak alaehGivfg EpKtdidkir twkEkvInqL TGGlagMaKg rkVkVVnGlg VPkKvMwNta vhsefMhDha ...dyGfpsc EgKfNwrvik ekrDayVsrL naiyqnnLtk shIeIIrGha VPSKiMiraa HiaHlrrEsp fdggiaatvp tIdrskllaq qqarvdelrh akyegiLggn paItVVhGea

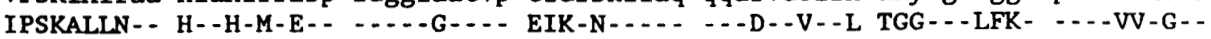

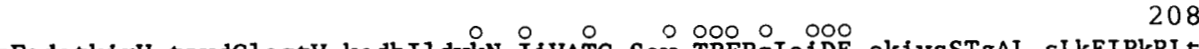
sFedetkirv tpvdGlegtV kedhIldvkN IIVATG. Sev TPFPgIeiDE ekivsSTgAL sLkEIPkRLt kFtgantleV egeng..... ...ktVinfdN aiIAaG.Srp iqLPfIphED priwdSTdAL eLkEVPeRL1 aF........ tsdpkptieV sgkkytaph. IlIATGgmps TPhesqipga slgitSdgfF qLeElPgRsv rFkddqsitv rlneGgerv mfdrcl.... . . VATG.asp avpPipg1kE spywtSTeAL asdtIPeRLa

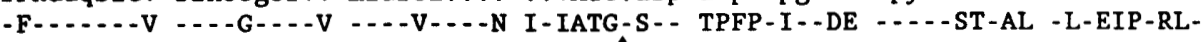

IIGgGIIGLE MgsVYsrLGS KVtVvefqpq IgasmDgeVa katqkflkkq gldFklstkv isakrnddkn

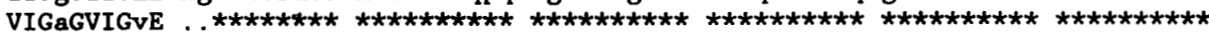
VmGgGIIGLE MgtVYhaLGS qIdVvemfdq VipaaDkdIv kvftkriskk fnlMletkvt aveakedgiy IVGaGyIavE MagILsaLGS Ktslmirhdk VlrsfDsmIs tncteelena gvevlkfsqv kevkktlsgl VIGssVVaLE LaqaFarLGS KVtVlarntl ffredpaige avtaafraeg levLehtqas qva......

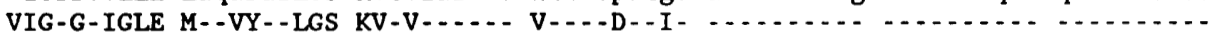

vveiv. .ved tktnkqenle aEvLLVAVGR rPyiagLgaE KiGlEVDkrG rlvIDdqFns kfPhIkvVGD

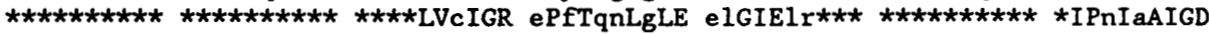
v.....tme gkkapaepqr yDavLVAIGR vPNgknLdag KaGVEVDdrG fIrVDkqLrT nVPhIFAIGD evsmvtavpg rlpvmtmipd vDcLLwAIGR vPNTkdLsLn KlGIqtDdkG hIIVDefqnT nVkgIYAVGD ...hmdgef vlttthgelr aDkLLVAtGR tPNTrsLaLD aaGVtVnaqG aIVIDqgMrT snPnIYAaGD - -D-LLVAIGR - PNT - - L-LE K-G-EVD - -G -IV-D- - - T -VP-IYA-GD

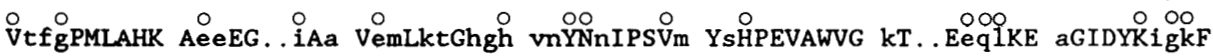
VvagPMLAHK AedEG..ilc VegMagGavh IDYNcVPSVI YTHPEVAWVG k..sEeq1KE eGIEYKvgkF IvgqPMIAHK gwhEGhvaAe Viagk. .khy FDpkvIPSIa YTePEVAWVG 1T..EkeaKE kGIsYetatF VcgkaLLtpv AiaaGrk1Ah rlfeykedsk LDYNnIPtVV FsHPpIgtVG 1TedEaihKy gienvktyst ctdqPqFvyv AaaaG..tra ainMtgGdaa LDltamPaVV FTdPqVAtVG ..ysEaeahh dGIEtdsrtL V-- PMLAHK A--EG---A- V--M--G--- LDYN-IPSVV YTHPEVAWVG -T--E---KE -GIEYK---F

482

PFAansRAkT 음ำ PFA***RAkT NaDTDGmVKi lgqkstDRV1 GaHIIGPgAG EMInEAALAl EyGAscEDIA rvcHAHPTLS PWAasgRAia sdcaDGmtKL IfDkeshRVI GgaIVGtngG ELlgEigLAI EmGcdaEDIA 1tiHAHPTLh sFtpmyhAvT krkTkcvmkM VcankeEkVV GiHmqGlgcd EMlqgfAvAV kmGAtkaDfd ntvaiHPTsS tLdnvpRAla NfDTrGfIKL ViEegshRII GvqaVaPeAG ELIqtAALAI rnrmtvqElA dq1fpylTMv PFA---RA-T N-DTDG-VKL V-D--ERVI G-HI-GP-AG EMI-EAALAI E-GA--EDIA - - -HAHPTLS

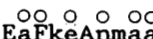

EaFkeAnmaa ydkaihc........

EaFreAnlaa sfgkainf.......

EsvglAaevf egsitdlpnp kakkk

EeLvt1r... ...........

EgLklAaqtf nkdvkqlscc ag...

E-... A.............. 
(a)

GCN4 binding site consensus sequence

$\begin{array}{ll}L P D 1 & -264 \\ L P D 1 & -247 \\ L P D 1 & -114\end{array}$

rrTGACTCatt $---t$

CGTGAATCGTTTTTAG GATGACTCGTTTTTAA

$-253$

$-236$

$\angle P D$

TTTGACTCACCTCGAA

$-108$

(b)

CYCI UAS1

CYC1 UAS2

$G A L 1-10$ control region sequence

LPDI

$-204$

CTCATTGGCGAGAAGTC

$-189$

(c)

Heat-shock consensus sequence

$$
\text { LPDI }
$$

C--GAA--TTC--G

\section{CACGAATAGTCATG}

Fig. 3. Alignment of potential regulatory sequences with known motifs. In each case the established regulatory sequence is displayed, with the upstream regions of the $L P D I$ gene which show homology shown directly below. (a) Consensus sequence for the general control regulatory site. Highly conserved nucleotides are shown as capitals, conserved residues are shown in lower case, and nonconserved residues are indicated by dashes. (b) Upstream activation sites (UAS1 and UAS2) of $C Y C 1$ and a sequence located in the GALI-10 control region. (c) Heat-shock promoter consensus sequence.

surprising than it seems at first sight, since some of the tricarboxylic acid (TCA) cycle intermediates are substrates for the synthesis of such amino acids as glutamate, arginine, lysine and proline. There is one report that fumarase activity is derepressed in arginine bradytrophs; it has been suggested that this TCA cycle enzyme may also be under general amino acid control (Delforge et al., 1975).

A second putative control region, at position -204 , together with its homology with the upstream activation sites of $C Y C 1$, is shown in Fig. 3. The function of this region is not known but both genes are involved in aerobic metabolism and are subject to catabolite repression (Guarente et al., 1984; Roy \& Dawes, 1987); a related sequence with homology over 8 bases can be found in the GALI-10 control region (Johnston \& Davis, 1984).

A third potential control site shown in Fig. 3 is the sequence at -352 which shows a 7 out of 8 match with the general heat-shock consensus sequence (Pelham, 1985).

Several further sequences are also of interest. One is an inverted repeat CTCCGCGGAG at position -188 which is similar to a general repressor protein binding sequence proposed by

Fig. 2. Protein sequence comparison. The primary structures of $S$. cerevisiae lipoamide dehydrogenase (YLPDH), pig heart lipoamide dehydrogenase (PLPDH), E. coli lipoamide dehydrogenase (ECLPDH), human red blood cell glutathione reductase (HGR) and Pseudomonas aeruginosa mercuric reductase (PAMR) are aligned for maximum homology. The residue numbering is based on $S$. cerevisiae lipoamide dehydrogenase. A consensus line below the five sequences shows residues common to three or more of the amino acid sequences. The borders of the domains representing the four regions of strong homology are based on glutathione reductase and are marked by arrowheads below the consensus sequence. The 80 residues at the $\mathrm{N}$-terminus of mercuric reductase have been omitted. The individual sequences show residues in agreement with the consensus sequence in upper case and all others in lower case. The alignment was performed using the program PRETTY with the default protein comparison file (Gribskov \& Burgess, 1986), which regards certain amino acids as similar for the purposes of the consensus line. The asterisks in the pig heart lipoamide dehydrogenase sequence indicate regions of unknown sequence. Residues common to pig heart and yeast lipoamide dehydrogenase but not to the $E$. coli lipoamide dehydrogenase are shown by circles above the yeast sequence. 
T. Cooper (personal communication); a second is the sequence CACCTCGA at position -109 which is homologous to one found upstream of the $A R G 3$ and $C A R I$ genes involved in arginine biosynthesis and degradation respectively (Crabeel et al., 1985). In addition to the above, the upstream region contains a number of direct repeats and inverted repeats which may also have a role in the regulation of $L P D 1$ expression. One of these at -283 is very interesting since it is a $23 \mathrm{bp}$ inverted repeat, containing direct repeats of $9 \mathrm{bp}$ each within it. It is represented in a symmetrically truncated form at -361 . We are currently investigating the functional significance of these upstream regions.

We are grateful to Lynn Kennedy for technical assistance, and to the SERC for a postgraduate studentship (J.R.) and research grant support.

\section{REFERENCES}

Crabeel, M., Huygen, R., Verschueren, K., MesSENGUY, F., Tinel, K., CUnin, R. \& GlansdorfF, N. (1985). General amino acid control and specific arginine repression in Saccharomyces cerevisiae: physical study of the bifunctional regulatory region of the ARG3 gene. Molecular and Cellular Biology 5 , 3139-3148.

Delforge, J., Messenguy, F. \& Wiame, J. (1975). The regulation of arginine biosynthesis in Saccharomyces cerevisiae. European Journal of Biochemistry 57, 231239.

De Marcucci, O. L., Hunter, A. \& Lindsay, J. G. (1985). Low immunogenicity of the common lipoamide dehydrogenase subunit (E3) of mammalian pyruvate dehydrogenase and 2-oxoglutarate dehydrogenase multienzyme complexes. Biochemical Journal 226, 509-517.

DevereuX, J., Haeberli, P. \& Smithies, O. (1984). A comprehensive set of sequence analysis programs for the VAX. Nucleic Acids Research 12, 387-395.

Dickinson, J. R., Roy, D. J. \& DAWEs, I. W. (1986). A mutation affecting lipoamide dehydrogenase, pyruvate dehydrogenase and 2-oxoglutarate dehydrogenase activities in Saccharomyces cerevisiae. Molecular and General Genetics 204, 103-107.

GrIBSKov, M. \& BURGESS, R. R. (1986). Sigma factors from $E$. coli, B. subtilis, phage SP01 and phage T4 are homologous proteins. Nucleic Acids Research 14, 6745-6763.

GrossChedL, R. \& BIRNSTIEL, M. L. (1980). Identification of regulatory sequences in the prelude sequences of an $\mathrm{H} 2 \mathrm{~A}$ histone gene by the study of specific deletion mutants in vivo. Proceedings of the National Academy of Sciences of the United States of America 77, 1432-1436.

Guarente, L., Lalonde, B., Gifford, P. \& Alani, E. (1984). Distinctly regulated tandem upstream activation sites mediate catabolite repression of the $C Y C 1$ gene of Saccharomyces cerevisiae. Cell 36, 503-511.

Guest, J. R. \& Creaghan, I. T. (1973). Gene-protein relationships of the $\alpha$-keto acid dehydrogenase complexes of Escherichia coli K12: isolation and characterization of lipoamide dehydrogenase mutants. Journal of General Microbiology 75, 197-210.

Guest, J. R. \& RicE, D. W. (1984). Molecular genetic approaches to the study of $E$. coli flavoproteins. In Flavins and Flavoproteins, pp. 111-123. Edited by R. C. Bray. Berlin: Walter de Gruyter. von HeIJNe, G. (1986). Mitochondrial targeting sequences may form amphiphilic helices. $E M B O$ Journal 5, 1335-1342.

Hill, D. E., Hope, I. A., Macke, J. P. \& Struhl, K. (1986). Saturation mutagenesis of the yeast his 3 regulatory site: requirements for transcriptional induction and for binding by GCN4 activator protein. Science 234, 451-457.

HUNTER, A. \& LiNDSAY, J. G. (1986). Immunological and biosynthetic studies on the mammalian 2oxoglutarate dehydrogenase multienzyme complex. European Journal of Biochemistry 155, 103-109.

Johnston, M. \& DAVIS, R. W. (1984). Sequences that regulate the divergent $G A L 1-G A L 10$ promoter in Saccharomyces cerevisiae. Molecular and Cellular Biology 4, 1440-1448.

KoCHI, H. \& KIKUCHI, G. (1976). Mechanism of reversible glycine cleavage reaction in Arthrobacter globiformis: function of lipoic acid in the cleavage and synthesis of glycine. Archives of Biochemistry and Biophysics 173, 71-81.

KozaK, M. (1981). Possible role of flanking nucleotides in recognition of the AUG initiation codon by eukaryotic ribosomes. Nucleic Acids Research 9 , 5233-5252.

Lawson, U. K., CoOK, K. G. \& Yeaman, S. J. (1983). Rapid purification of bovine kidney branched-chain 2-oxoacid dehydrogenase complex containing endogenous kinase activity. FEBS Letters 157, 54-58.

Maniatis, T., FRITSCH, E. F. \& SAMBroOK, J. (1982). Molecular Cloning: a Laboratory Manual. Cold Spring Harbor, NY: Cold Spring Harbor Laboratory.

MESSING, J. (1983). New M13 vectors for cloning. Methods in Enzymology 101, 20-78.

Mukherjee, B. B., Matthews, J., Horney, O. L. \& REED, L. J. (1965). Resolution and reconstitution of Escherichia coli $\alpha$-ketoglutarate dehydrogenase complex. Journal of Biological Chemistry 240, 2268-2269.

Pelham, H. (1985). Activation of heat shock genes in eukaryotes. Trends in Genetics 1, 31-35.

Proudfoot, N. J. \& Brownlee, G. G. (1976). 3' noncoding region sequences in eukaryotic messenger RNA. Nature, London 263, 211-214.

ReED, L. J. (1974). Multienzyme complexes. Accounts in Chemical Research 7, 40-46.

Robinson, J. R., Klein, S. M. \& SAgers, R. D. (1973). Glycine metabolism. Lipoic acid as the prosthetic 
group in the electron transfer protein $\mathbf{P}^{2}$ from Peptococcus glycinophilus. Journal of Biological Chemistry 248, 5319-5323.

RoY, D. J. \& DAwES, I. W. (1987). Cloning and characterization of the gene encoding lipoamide dehydrogenase in Saccharomyces cerevisiae. Journal of General Microbiology 133, 925-933.

Sanger, F., Nicklen, S. \& Coulsen, A. R. (1977). DNA sequencing with chain terminating inhibitors. Proceedings of the National Academy of Sciences of the United States of America 74, 5463-5467.

SMITH, H. O. (1980). Recovery of DNA from gels. Methods in Enzymology 65, 371-380.

SPENCER, M. E. \& GUEST, J. R. (1985). Transcriptional analysis of the sucAB aceEF and lpd genes of E. coli. Molecular and General Genetics 200, 145-154.

Stephens, P. E., Lewis, H. M., Darlison, M. G. \& GuEST, J. R. (1983). Nucleotide sequence of the lipoamide dehydrogenase gene of Escherichia coli K12. European Journal of Biochemistry 135, 519-527.
STRUHL, K. (1982). Regulatory sites for HIS3 expression in yeast. Nature, London 300, 284.

WIELAND, O. H. (1983). The mammalian pyruvate dehydrogenase complex: structure and regulation. Physiological and Biochemical Pharmacology 96, 123170.

Williams, C. H. JR, Arscott, L. D. \& Swenson, P. (1984). Active site chemical modification and sequencing of flavoproteins. In Flavins and Flavoproteins, pp. 95-109. Edited by R. C. Bray. Berlin: Walter de Gruyter.

WINTER, G. \& FIELDS, S. (1980). Cloning of influenza cDNA into M13: the sequence of the RNA segment encoding the A/PR/8/34 matrix protein. Nucleic Acids Research 8, 1965-1974.

ZareT, K. S. \& Sherman, F. (1982). DNA sequences required for efficient transcription termination in yeast. Cell 28, 563-573. 